In: M. Bitbol et al. (eds.): "Constituting Objectivity: Transcendental Perspectives on Modern Physics". Springer, Berlin. In preparation. (Version from July 2006, small corrections March/December 2007).

\title{
Structural Realism and Abductive-Transcendental Arguments
}

\author{
Holger Lyre \\ Philosophy Department, \\ University of Bonn ${ }^{1}$
}

\begin{abstract}
$\underline{\text { Abstract }}$
The paper deals with an attempt to present an "abductive-transcendental" argument in favour of a particular version of structural realism (SR), dubbed Intermediate SR. In the first part of the paper the general structure of transcendental arguments is scrutinized with a close view on Kant's original version and the prospect of their abductive variation. Then the role of symmetries in modern physics, especially symmetries without real instantiations and in particular gauge symmetries is discussed. This is combined with a presentation of SR as a promising current version of scientific realism. The discussion is supported by various arguments form gauge theories in modern physics. Intermediate SR, a realist position about all and only structurally derivable entities located between the extremes of Epistemic and Ontic SR turns out as the best fit to our current fundamental gauge physics and this finally leads to an abductive-transcendental reasoning concerning this position.
\end{abstract}

\section{Transcendental and Abductive-transcendental Arguments}

It seems to be a clear lesson from the history of modern physics, that the Kantian program - taken literally - is wrong-headed. Kant's way of deriving Newtonian physics in his Metaphysical Foundations of Natural Science (1786) seems evidence enough that his way of deriving the fundamental laws of physics is fatally flawed. Why, then, should one be interested in the Kantian program at all? Indeed a reason could be that if we strip off the inclinations of armchair philosophy from Kant's program, there might remain a core which could be still of value in modern science. This core, the core of the whole Kantian enterprise, survives in the structure and nature of transcendental arguments on which we will focus in the following.

Kant introduces transcendental arguments as arguments referring to the very preconditions of experience. In Critique B 25 he writes:

"I call all cognition transcendental that is occupied not so much with objects but rather with our mode of cognition of these objects insofar as this is to be possible a priori." [p. 149]

And in B 80 we find:

"...that not every a priori cognition must be called transcendental, but only that by means of which we cognize that and how certain representations (intuitions or conceptions) are applied entirely a priori, or are possible (i.e., the possibility of cognition or its use a priori).” [p. 196]

1Email: lyre@uni-bonn.de 
Hence, transcendental knowledge is meta-knowledge about how a priori knowledge is used. As such, transcendental knowledge is itself a priori knowledge. We must therefore first clarify our understanding of the a priori. One of the difficulties with Kant is that in his formulations he invites us to misinterpret the way in which his account leads to a subject-relatedness of knowledge. We may very well understand his talk about "modes of cognition" and "representations" as talk about the particular human cognitive machinery. And Kant endorses such an understanding for instance in passages like A 26-27 about the a priori character of space:

"We can therefore speak of space, extended objects, and so on, only from the buman standpoint ... For we cannot judge at all whether the intuitions of other thinking beings are bound to the same conditions that limit our intuition and that are universally valid for us.” [p. 160]

From a general epistemological perspective, however, it is certainly not necessary to restrict the a priori forms of cognition especially to humans. On the contrary: in B 72 for instance Kant, too, adds that

"It is also not necessary for us to limit the kind of intuition in space and time to the sensibility of buman beings; it may well be that all finite thinking beings must necessarily agree with human beings in this regard (though we cannot decide this)...”[p. 191]

Kant's point is in fact far better made if we think of the preconditions of experience - manifested in the a priori forms of cognition - as entirely general methodological preconditions. Call this the generality claim. It is the claim that the results of the transcendental method aren't at all bound to human experience or cognition in particular, but rather to experience or cognition in general. And that they therefore apply to any cognitive system or agent with "empirical competence."

While it is perhaps not entirely clear whether Kant himself definitely subscribes to the generality claim, he nevertheless subscribes to a further feature of his enterprise, which is in fact a natural consequence of the generality claim. This feature is the view of the a priori as being no part of the cognitive machinery itself, or, to put it bluntly, the view that the a priori forms of cognition are not themselves instantiated in terms of the mind's hardware. Surely, Kant falls back on a whole variety of capacities of the mind such as intuition, understanding, faculty of judgement or the like to spell out his aprioristic approach, but simultaneously he explicitly denies that ideas a priori - neither pure intuitions nor categories - are innate (see for instance the „Streitschrift gegen Eberhard“, 1790). Speaking in more modern terms, the a priori forms are not subject to empirical cognitive science, as also pointed out in the Critique B 78:

[Transcendental logic...] "has no empirical principles, thus it draws nothing from psychology (as one has occasionally been persuaded), which therefore has no influence at all on the canon of the understanding. It is a proven doctrine, and everything in it must be completely a priori." [p. 195]

Thus, Kant subscribes to a mind-dependency of our knowledge in a rather indirect sense only. What he intends is a methodological understanding of the a priori: It is, for instance, a methodological requirement to bring the manifold of sense data into a temporal one after another, a spatial side by side, or a causal connectedness. It is the general structure of a transcendental argument from which the justification of the doctrine of such a methodological a priori can be drawn.

What, now, is the general structure of a transcendental argument? An informal version following Kant would be that the possibility of experience $(\mathrm{E})$ demands the very preconditions of experience (PE) to hold. By reading this in the sense that the fact of E implies PE, one would make the argument an instance of modus ponens:

$\mathrm{E}$

$\mathrm{E}-->\mathrm{PE}$

PE 
The major drawback of this reading, however, is that this doesn't render transcendental arguments as synthetic a priori, for neither the first nor the second premiss of the above conclusion is synthetic a priori. The first premiss, the mere fact that $\mathrm{E}$, is contingent and thus synthetic a posteriori, whereas the second premiss represents an analytic statement. Obviously, the transcendental argument structure cannot be captured in a strict logical way, but should perhaps rather be reconstructed as an inference to the best explanation: the existence and validity of PE is the most plausible explanation for $\mathrm{E}$.

Of course, the reconstruction of transcendental arguments as inferences to the best explanation heavily undermines Kant's own far more rigorous understanding of his enterprise. For it is Kant's special claim that synthetic judgements a priori are accompanied by necessity and generality. But it is exactly this demand, which should better be weakened in view of a modern revised use of transcendental arguments, and indeed in two senses: As the best explanation for $\mathrm{E}, \mathrm{PE}$ is only very likely, but not necessary. Further on, the contingency of $\mathrm{E}$ as a premiss indicates that the conclusion doesn't yield in any possible world, but only in E-worlds, worlds in which experience takes place. We might therefore introduce the term abductive-transcendental argument for any inference to the most plausible and obvious preconditions of experience.

As a further digression from Kant's original approach, mention should be made that the introduction of the thing-in-itself isn't a necessary consequence of the transcendentally motivated methodological a priori. It is widely believed that perhaps the most urgent reason for Kant to introduce the thing-in-itself doesn't lie in his transcendental epistemology as such, but rather in the overall architecture of his philosophical system - in particular his account of free will beyond the dichotomy of compatibilism and non-compatibilism. Kant saw it as an explanation how we can have strict causal determinism in the realm of theoretical philosophy, where we are dealing with the things as phenomena, and at the same time the freedom of will within the realm of practical philosophy, where we are bound to the idea of absolute freedom.

To sum up: neither the general structure of an abductive-transcendental argument nor the methodological nature of the a priori depends on

- the existence of a particular, perhaps innate structure of our cognitive ability,

- necessity in the strong sense of being the case in all possible worlds,

- the existence of epistemically inaccessible things-in-themselves.

Remarkably, perhaps, such an understanding of a methodological a priori is in principle also in tune with a naturalistic picture of the world. Transcendental arguments impose constraints on the constitution of the knowledgeable world and possess insofar a certain normative character. Construed as abductive-transcendental arguments, however, that is as inferences to the best explanation, such normativity doesn't come equipped with absolute necessity.

\section{Physical symmetries without real instantiations}

From a transcendental meta-perspective about science we now shift to modern science directly, in particular to the role of symmetries. Symmetries are the hallmark of modern physics - and they come in many different varieties: we distinguish continuous from discrete, global from local, active from passive, geometrical from dynamical, and so on. Given the preeminent role symmetries play in contemporary physics, it is more than natural to ask for a deeper reason for their omnipresence. One such reason can be seen in the quite general characterization which can be given to symmetries as an overall topos: they seem to provide a formal device for the general interplay between change - captured in terms of symmetry transformations - and persistence - the symmetry invariants. Let us follow this general theme in some more detail. 
Given any domain D (usually the state space of the physical system), a symmetry of D consists in a set of one-to-one mappings, the symmetry transformations, of D onto itself, such that the structure of $\mathrm{D}$ is preserved. The structure of $\mathrm{D}$ can be understood as a set of relations imposed on D. It can then easily be seen that the symmetry transformations of $\mathrm{D}$ form a group and simultaneously exemplify equivalence relations and, hence, induce a partitioning of $\mathrm{D}$ into equivalence classes. It becomes clear from such a characterization that symmetries can be used as tools to filter out specific structural aspects. Moreover, since the set of all symmetries of a domain D is sufficient to characterize the entire structure of $\mathrm{D}$ and if structural aspects provide the relevant aspects of a given domain, symmetries may very well work as filters for relevance.

Consider the simple example of the (cyclic) group of rotations of a square. We get 4 rotations around 90, 180 and 270 degrees as well as the identity mapping with 0 or 360 respectively. These four rotations $R_{1}$ to $R_{4}$ form a group $G$, that is any combined transformation $R_{i} \times R_{j}$ can be mapped onto one of the elements of G. $G$ is the group of isometries of the square, that is the group of actions which preserve and characterize the "relevant structure" of the square as a geometrical figure.

A further distinction, not yet mentioned, is definitely important for modern physics: the distinction between symmetry transformations with or without real instantiations. The example just given is obviously a symmetry for which real instantiations in terms of rotations of squared corporeal objects in the world can be considered. Quite generally, space-time transformations instantiated in terms of rotations, translations or boosts of reference frames are paradigmatic cases of symmetries in physics with real instantiations. By way of contrast, symmetries without real instantiations include for instance scale transformations, coordinate transformations or, what will be our particular concern, gauge transformations.

One might in fact wonder why symmetries without real instantiations are of any value in physics at all. Apparently, such symmetries reflect a redundancy in the description of a system. Striving for nonredundancy such symmetries seem, on a rigorous level, to be superfluous. At the same time, however, the merit of symmetries about superfluous descriptive structure may nevertheless lie in their way of highlighting certain relevant structural invariants. In this sense symmetries without real instantiations fall precisely under the class of "filters for relevance."

Gauge theories in modern physics, that is theories which are characterised by their respective gauge symmetries, are most prominently displayed in the Yang-Mills theories of the Standard Model and represent the most important case of an application of symmetries as filters for relevance. Within the Lagrangian approach, the common mathematical framework of the practising high energy physicist, a gauge theory is mainly represented by a Lagrangian $\mathfrak{L}$ built out of three terms: the kinetic part $\mathfrak{L}_{\text {kin }}$, the gauge field Lagrangian $\mathfrak{L}_{\mathrm{f}}$ and the interaction term $\mathfrak{L}_{\text {int }}$. In Standard Model gauge theories, $\mathfrak{L}$ shows a symmetry under both global and local gauge transformations, that is transformations of the Dirac wave spinor which represents the fundamental fermionic matter fields and which is governed by $\mathfrak{L}_{\text {kin }}$. The requirement to formulate a respective gauge theory in the most general gauge covariant representation, that is to represent the theory in such a way that no dependency from the obviously non-instantiable gauge transformations exists (both global and local), is usually known as the gauge principle. This requirement is fulfilled on the Lagrangian level if $\mathfrak{L}_{\text {kin }}$ is enlarged to $\mathfrak{L}_{\text {kin }}+\mathfrak{L}_{\text {int }}$, where $\mathfrak{L}_{\text {int }}$ includes an inhomogeneous term - basically the gradient of the phase of the wave function - needed to satisfy the gauge symmetry requirement. As such, $\mathfrak{L}_{\text {kin }}$ has no further physical significance (as sometimes mistakenly stated in parts of the textbook literature), the structure of $\mathfrak{L}_{\text {kin }}$, however, does of course reflect the particular gauge symmetry. Technically speaking, $\mathfrak{L}_{\text {kin }}$ mainly includes the connection field of a principal fiber bundle, the appropriate geometrical structure to describe a Lagrangian gauge theory. 
The connection has values in the Lie algebra of the Lie structure group of the bundle, the physically considered gauge group (for a detailed presentation see for instance Lyre 2004a, b).

The gauge symmetry group not only determines the connection, physically identified as the gauge potential, but also the derivative of the potential, the gauge field tensor (out of which $\mathfrak{L}_{\mathrm{f}}$ is built). While the gauge potential isn't a directly observable, i.e. measurable, quantity, the gauge field strength is. Whether a particular gauge field is realized in nature is of course an empirical question, its mathematical characterization can however simply be given by the gauge group under which the gauge tensor, technically the curvature tensor of the bundle, is preserved. Due to the Standard Model, the groups $\mathrm{U}(1), \mathrm{SU}(2)$ and $\mathrm{SU}(3)$ refer to gauge fields with real instantiations. Again, the field tensors are invariants under the respective gauge symmetries, meaning that the gauge transformations themselves have no physical instantiations. In principle only the gauge invariants allow for a realistic interpretation (though this does not necessarily mean that the gauge invariance of a quantity is sufficient for its observability).

\section{Two strands of Structural Realism}

It is now time to introduce Structural Realism (SR). Broadly construed, SR is the doctrine that scientific realists should be committed to the structural content of our best theories rather than its content in terms of object-like entities. The account has its forerunners in such eminent thinkers like Poincaré, Eddington, Weyl on the physicists side as well as Cassirer, Schlick, Russell and Quine on the side of philosophy. The recent debate decomposes into two strands. John Worrall (1989) recommended SR as an appropriate means to make capital out of the prospects of realism, notably its straightforward explanation of the success of science without making it a miracle (the "no miracles argument"), while simultaneously escaping the two most dominant anti-realistic arguments: the pessimistic meta-induction (PMI) and theory underdetermination (TUD). Let us call this the Worrall-type SR, based on arguments from the general philosophy of science, in particular arguments about the pros and cons of scientific realism. The second strand was mainly inaugurated by the work of Steven French (1998) and James Ladyman (1998; French and Ladyman 2003) and here the idea is that arguments in favour of SR are derived directly from an ontological analysis of the particular sciences, notably modern physics. Call this the French-Ladyman-type SR, to which we will focus in a moment.

But let us, ever so briefly, first consider Worrall-type SR. Confronted with PMI, SR proponents point out that the structural aspects show a far greater continuity through theory change than the notorious turn overs of theoretical terms referring to supposed object-like entities. A nice little example can be given from the early history of gauge theories. Classical electromagnetism shows a well-known gauge freedom in the potential-formulation under a group isomorphic to the real numbers. Quantum electrodynamics, the historical the successor, is properly understood as a $U(1)$ gauge theory with an underlying fiber bundle structure, where the potentials represent connection fields which again take on values in the reals, now considered as the Lie algebra of the bundle's structure group, the gauge group $\mathrm{U}(1)$. The group and invariance structure of the former theory has thus been embedded into the larger mathematical structure of the successor theory.

Surely not every progress in science shows such an elegant retention of structure through theory change. In order to avoid the force of PMI, one has to come up with an at least half-convincing reconstruction of the history of scientific progress in terms of an entirely structural characterization of theories. And this, of course, is an open research project, albeit certain steps in this direction are in fact already done (one such step is the work of Scheibe 1997/1999 about theory reduction within a fairly structural approach). Nevertheless, it seems quite reasonable to try to circumvent the anti-realistic threat of PMI from a structuralist perspective, and likewise the threat of TUD. By not committing us to an ontology of objects, SR proponents claim, we are able to escape the usual underdetermination scenarios. For there is a far greater similarity between theory alternatives or rivalling theoretical 
frameworks regarding their structural content as opposed to their entity content. Of course it is not clear whether TUD as a general threat is always limited to rival entities as opposed to structure. But given the fact that convincing cases of TUD (i.e. no fancy artificial or pathological examples) within the practising sciences are seldom to be found anyhow, a convincing case of structural incompatibility of two otherwise empirically indistinguishable theories is indeed not in sight.

For TUD, too, a nice gauge theoretic example can be given: Gauge theories comprise so-called holonomy and non-trivial topological effects. Regarding the former the Aharonov-Bohm (AB) effect is a case at hand. In this type of effect the gauge field seems to interact non-locally with the matter field wave function, thereby violating both the principle of local action and the concept of point-like interaction. In order to avoid such non-locality an explanation in terms of gauge potentials can be given at the metaphysical price of giving up separability and observability of the underlying entities while at the same time retaining local action and point-like interaction. A third interpretation is also feasible in terms of holonomies or Wilson loops as basic entities (that is loop integrals of the potential), which violate separability and point-like interaction, but are gauge invariant and, hence, observable. The metaphysical question about the underlying basic entities of gauge theories (fields, potentials or holonomies) is thus underdetermined by empirical evidence. SR, however, has a straightforward response at hand: it is precisely the gauge group (as well as the entire fiber bundle structure) which is invariant in all three rivalling interpretations. The anti-realistic threat of TUD vanishes once our realistic commitment sticks with the group structure only (cf. Lyre 2004a, b).

But, as mentioned, such "indirect" Worrall-type evidence for SR isn't the only game in town. A more direct proof for SR's metaphysical picture of the world would be far more significant. This is exactly the more recent strategy of the French-Ladyman-type of arguments in favour of SR. And although it is certainly not possible to read off one's metaphysics from physics in a naïve manner, it might very well be possible to draw certain consequences from an ontological analysis. One such consequence could be the "metaphysical underdetermination" ascribed to quantum theory by French (1998). He argues that quantum theory is plagued by an underdetermination of a very special kind which affects even the core metaphysical concept of individuality itself - more precisely the question whether quantum particle indistinguishability leaves us with non-individuals or rather some form of primitive thisness or haecceity. And indeed a rather similar scenario applies to the metaphysics of spacetime points, where diffeomorphism invariance plays the analogous role to permutation invariance in quantum theory (cf. Stachel 2002).

In view of the above mentioned TUD scenario of gauge theories we may as well state that insofar as the notions of locality and separability are underdetermined in their application and hence the spatiotemporal nature of our physics' most fundamental entities as well as the doctrine of Humean supervenience are left undecided, again a type of underdetermination occurred which affects genuine and deep metaphysical concepts - and which therefore suggests in a more direct way to give up any realistic commitment according object-like entities and to stick with the symmetry structure alone. So besides quantum theory and general relativity we also find French-Ladyman-type of arguments for SR in the field of gauge theories. Indeed, an increasing community of authors within recent years has joined the new strand of SR including Tian Yu Cao, Mauro Dorato, Michael Esfeld, Michael Redhead, Dean Rickles, Simon Saunders and Howard Stein, to mention just a few.

\section{Intermediate Structural Realism}

James Ladyman (1998) introduced another distinction between two kinds or variants of SR: an epistemic (ESR) and an ontic version (OSR). His idea was to combine the French-Ladyman-type of arguments for SR in particular with OSR. There is, however, no immediate reason for this parallel. While it may be the case that Worrall-type SR is more naturally in tune with ESR - the view that structures as well as the bearers of the structure exist, but that such bearers are epistemically 
inaccessible to us - no such immediate connection between French-Ladyman-type arguments and OSR - the radical view that, as the slogan goes, "structures is all there is" - exists. It may instead very well be the case that authors who prefer to consider French-Ladyman-type of arguments for SR, will nevertheless reject OSR.

Michael Esfeld (2004), for instance, argues for a version of SR he calls moderate SR. But at the same time, Esfeld joins company with the majority of both defenders and critics of SR who feel uneasy - to say the least - with the radical idea of OSR, which seems to suggest that if it is only structures that exist and if structures are really just sets of relations, but that such relations need certain relata as their very constituents, then the doctrine of OSR seems to be a doctrine about relations without relata - and that is a logical inconsistency. Instead, what is quite possible and according to Esfeld appropriate to quantum theory (with its tensor product structure of Hilbert state spaces which lead to correlations and entangled states) is a pure ontology of relations, such that the relata exist but are individuated only through their structural and, hence, relational properties. Esfeld therefore seeks to reject the idea of intrinsic properties on the fundamental level.

A further difficulty, however, arises here as well. As we have seen above, the overall topos of symmetries is their handling of the quite general concepts of change and persistence. This is in particular true for the symmetries of physical state spaces such as the Hilbert space of a quantum system. The symmetries of the state space highlight its structural features as invariants under the symmetry transformations. Such invariants, however, comprise intrinsic properties of physical object-systems. But Esfeld's moderate SR focuses exclusively on state-dependent properties connected with the self-adjoint operators on a Hilbert space such as spatial position or particular spin directions. Inevitably, however, via its symmetries, any state space will at the same time also comprise state-independent, intrinsic properties - on the fundamental level all and those properties which are given by the invariants under the Poincaré and various gauge groups, i.e. mass, spin and the various charges.

It is therefore implausible to seek for a pure metaphysics of relations - and this is all the more so if we are in search for a structurally declared metaphysics build on symmetries. State-independent, intrinsic properties are a necessary consequence of this view. And this is particularly important in case of the invariants of a state space under symmetry transformations without real instantiations such as gauge transformations. In this case a fortiori only the symmetry invariants can have a certain physical significance.

Nevertheless, two further limiting remarks about the nature of the symmetry invariance properties are in order. First, such properties are, as we may say, still structurally defined or derived. That is, their ontological status is secondary in contrast to the symmetry structure which is primary. And second: Invariance properties are not sufficient for an individuation of objects. They merely allow for the determination of object classes - no essentialism has been invoked. Let us call the view about SR thus developed "Intermediate SR" - a position between ESR and OSR. Its connection to ESR is that the relata are not denied, its OSR connection is that the relata are, however, only structurally defined in the above sense.

\section{Structural Realism and Abductive-Transcendental Analysis}

It has sometimes been claimed in the literature that ESR is a variant of Kantianism (cf. French and Ladyman 2003). Taken literally, this claim is of course nonsense, but a certain family resemblance stems from the fact that ESR denies access to the intrinsic natures of the things - and this echoes the Kantian thing-in-itself. But Kant's program has of course far wider scope not invoked by ESR. It is a merging of transcendental idealism and empirical realism and, according to the former, the Kantian view refers to structures as transcendental structures of knowledge or cognition (from which the transcendental subject can be determined), whereas according to the latter it shows no realistic preference for 
structures over objects. Moreover, SR, as a branch of scientific realism, concerns the status of theoretical terms rather than common-sense reality, whereas Kant considers reality in toto. So the claim should not be that any version of SR is literally a variant of Kantianism, but nevertheless the attempt shall now be made to collect the previous results together and to sketch the structure of an abductivetranscendental argument in favour of SR, more particularly ISR.

The first premiss is about the notion of experience: Experience means to recognize the change of the variable in relation to the permanent or persistent. Take this as an analytic statement about experience and, hence, empirical science. Experience would be impossible in both a world of absolute change or absolute persistence.

This relates to the second premiss: Symmetry is the general mathematical tool to analyze the interplay between change and persistence. Any empirical science must allow, at its bottom level, a law-like description of the world in terms of symmetry structures.

Thirdly: ISR is the minimal doctrine satisfying a realist commitment to empirical science. This is of course not to say that ISR is the only reasonable realist doctrine (which would be an absurd armchair verdict), but that it is minimal as regards a science of structurally defined entities.

From this it follows that ISR is most plausibly the core of any realist position of fundamental empirical science. Here again the conclusion only presents ISR as a core position which must perhaps eventually be embedded in a wider position in the sense that more than structurally defined entities constituted by relational properties and intrinsic invariance properties are taken into account. The assumption, however, is that Occam's razor (a further tacit premiss in the above argument) cuts in favour of ISR. Hence, as an inference from the most basic premisses about empirical science to the best realist explanation of its successs today, ISR turns out the most plausible and natural candidate. 


\section{References}

M. Esfeld (2004): Quantum entanglement and a metaphysics of relations. Studies in History and Philosophy of Modern Physics 35:4, 601-617.

S. French (1998): On the Withering Away of Physical Objects. In: Interpreting Bodies: Classical and Quantum Objects in Modern Physics, ed. by E. Castellani. Princeton: Princeton University Press, pp. 93-113.

S. French and J. Ladyman (2003): Remodelling Structural Realism: Quantum Physics and the Metaphysics of Structure. Synthese, 136(1): 31-56.

B. Gower (2000): Cassirer, Schlick and 'Structural' Realism: The Philosophy of the Exact Sciences in the Background to Early Logical Empiricism. British Journal for the History of Science, 8: 71-106.

J. Ladyman (1998): What is Structural Realism? Studies in History and Philosophy of Science, 29(3): 409424.

H. Lyre (2004a): Lokale Symmetrien und Wirklichkeit. Mentis, Paderborn.

H. Lyre (2004b): Holism and Structuralism in U(1) Gange Theory. Studies in History and Philosophy of Modern Physics 35:4, 643-670.

I. Kant. Kritik der reinen Vernunft. Riga, 1781 (B edition 1787). [Kant quotes and pages refer to: P. Guyer and A. W. Wood (eds.), I. Kant: Critique of Pure Reason. Cambridge University Press, 1998].

I. Kant. Über eine Entdeckung nach der alle neue Kritik der reinen Vernunft durch eine ältere entbehrlich gemacht werden soll (,,Streitschrift gegen Eberhard“). 1790.

E. Scheibe (1997/1999): Die Reduktion physikalischer Theorien. 2 volumes, Springer, Berlin.

J. Stachel (2002): 'The Relations between Things' versus 'The Things between Relations': The Deeper Meaning of the Hole Argument. In: D. Malament (ed.)., Reading Natural Philosophy: Essays in the History and Philosophy of Science and Mathematics. Open Court, La Salle, Illinois.

J. Worrall (1989): Structural Realism: The Best of Both Worlds? Dialectica, 43: 99-124. 